\title{
Literaturverzeichnis
}

\section{Kommentare, Bücher}

\section{Baumbach/Hefermebl/Casper}

Wechselgesetz, Scheckgesetz, Recht des Zahlungsverkehrs: WG, ScheckG, Recht des Zahlungsverkehrs, Kommentar, 24. Aufl., 2020

\section{Böttcher}

Kommentar zum Zwangsversteigerungsgesetz, 6. Aufl., 2016 (zit.: Böttcher, ZVG)

Brox/Walke

Zwangsvollstreckungsrecht, 11. Aufl., 2018

\section{Bülow}

Wechselgesetz, Scheckgesetz mit AGB-Sparkassen, AGB-Banken, AGBPostbank und Scheckbedingungen, Kommentar, 5. Aufl., 2013

(zit.: Bülow, WechselG)

$B u \beta$

Das Nacherbenrecht in der Immobiliarzwangsversteigerung

(Osnabrücker Rechtswissenschaftliche Abhandlungen) 2004, S. $48 \mathrm{ff}$.

Dassler/Schiffhauer/Hintzen/Engels/Rellermeyer

Kommentar zum Zwangsversteigerungsgesetz, 16. Aufl., 2020

(zit.: D/S/H/E/R-Bearbeiter, ZVG)

\section{Depré}

Kommentar zum Zwangsversteigerungsgesetz, 2. Aufl. 2019

(zit.: Depré-Bearbeiter, ZVG)

Gladenbeck/Sambat

Kreditsicherung durch Grundschulden, 10. Aufl., 2020

\section{Haarmeyer/Hintzen}

Handbuch zur Zwangsverwaltung, 3. Aufl., 2012

(zit.: Haarmeyer/Hintzen, Handbuch zur Zwangsverwaltung)

\section{Haarmeyer/Hintzen}

Zwangsverwaltung, Kommentar, 6. Aufl., 2016

Zwangsversteigerungsgesetz ( $\mathbb{S} 146-161,172,173 \mathrm{ZVG})$

und Zwangsverwalterverordnung $\left(\mathrm{ZwVwV}_{\mathrm{w}}\right)$

(zit.: Haarmeyer/Hintzen, Zwangsverwaltung)

\section{Hartmann/Metzenmacher}

Umsatzsteuergesetz, Kommentar, Loseblattwerk, Stand April 2020 (zit.: Hartmann/Metzenmacher-Bearbeiter, UStG) 


\section{Hintzen}

Vollstreckung und Insolvenz, Kölner Schrift zur Insolvenzordnung,

2. Aufl., 2000

\section{Jezerwsi}

Eintragungen in Abteilung II des Grundbuches, 15. Aufl., 2017

Kirchbof

Insolvenzrecht in der Praxis Leitfaden zum Insolvenzrecht, 1999

Münchener Kommentar zum Bürgerlichen Gesetzbuch

hrsg. von Säcker/Rixecker/Oetker

Bd 1: Einleitung und Allgemeiner Teil, $\$ \int S 1-240$, AllgPersönlR, ProstG, AGG, 8. Aufl., 2018

Bd 8: Sachenrecht, $\iint 854-1296$, WEG, ErbbauRG

8. Aufl., 2020

(zit.: Bearbeiter, in: MünchKomm-BGB)

Münchener Kommentar zum Versicherungsvertragsgesetz

hrsg. von Langheid/Wand

Bd. 2, SS 100-216, 2. Aufl., 2017

(zit.: Bearbeiter, in: MünchKomm-VVG)

Münchener Kommentar zur Insolvenzordnung hrsg. von Stürner/Eidenmüller/Schoppmeyer

Bd. 2, SS 80-216, 4. Aufl., 2019

(zit.: Bearbeiter, in: MünchKomm-InsO)

Münchener Kommentar zur Zivilprozessordnung

hrsg. von Krüger/Rauscher

Bd 2, \S 355-945b, 5. Aufl., 2016

(zit.: Bearbeiter, in: MünchKomm, ZPO)

Musielak

Kommentar zur Zivilprozessordnung, 17. Aufl., 2020

(zit.: Musielak-Bearbeiter, ZPO)

Obermüller

Insolvenzrecht in der Bankpraxis, 9. Aufl., 2016

Prölss/Martin

Kommentar zum Versicherungsvertragsgesetz, 30. Aufl., 2018

(zit.: Prölss/Martin-Bearbeiter, VVG)

Rau/Dürrwächter

Kommentar zum Umsatzsteuergesetz Loseblattwerk, Stand März 2018

(zit.: Rau/Dürrwächter-Bearbeiter, UStG)

Stöber

Kommentar zum Zwangsversteigerungsgesetz, 22. Aufl., 2019

(zit.: Stöber, ZVG) 


\section{Stöber}

ZVG-Handbuch, Zwangsvollstreckung in das unbewegliche Vermögen,

9. Aufl., 2010 (zit.: Stöber, ZVG-Handbuch)

\section{Stöber/Rellermeyer}

Forderungspfändung - Zwangsvollstreckung in Forderungen

und andere Vermögensrechte, Handbuch, 17. Aufl., 2020

(zit.: Stöber/Rellermeyer, Forderungspfändung)

\section{Storz/Kiderlen}

Praxis des Zwangsversteigerungsverfahrens, 13. Aufl., 2020

Zöller

Kommentar zur Zivilprozessordnung, 33. Aufl., 2020

(zit.: Zöller-Bearbeiter, ZPO)

\section{Aufsätze}

\section{Abramenko}

Die Auferstehung einer Toten? Die Mietvorauszahlung nach Abschaffung $\operatorname{der} \iint 57 c, d$ ZVG, ZfIR 2014, 833

\section{Achenbach}

Zwangsversteigerung mehrerer Grundstücke. Probleme im Rahmen der

Zuteilung einer weiteren/bedingten Teilungsmasse, ZfIR 2019, 261

\section{Achenbach}

Zwangsversteigerung: Maßgeblicher Zeitpunkt für Anmeldungen und Folgen der Versäumung, ZfIR 2018, 440

\section{Alff}

Klauselprobleme in der Immobiliarvollstreckungspraxis, Rpfleger 2001, 385 Alff

Zur Pflicht des Gläubigers, nicht valutierte Zinsen für die Zeit nach Zuschlag aus einer vom Ersteher übernommenen Grundschuld geltend zu machen, Rpfleger 2011, 357

Alff/Hintzen

Hausgelder in der Zwangsversteigerung und Zwangsverwaltung,

Rpfleger 2008, 165

\section{Alff/Hintzen}

Kautionsrückzahlungspflicht des Zwangsverwalters, Rpfleger 2003, 635

\section{Becker}

Insolvenz des Grundstückseigentümers nach Anordnung der Zwangsversteigerung, ZfIR 2017, 813

Bergsdorf

Die Hotelimmobilie in der Zwangsverwaltung, ZfIR 2014, 842 


\section{Bergsdorf/Thrum}

Vorausverfügungen in der Zwangsverwaltung, ZfIR 2007, 164

\section{Bleutge}

Immobilienbewertung in der Zwangsversteigerung - Qualifizierte

Sachverständige sind unverzichtbar, ZfIR 2017, 52

\section{Böbringer}

Rechtsprobleme bei der Eintragung von Zwangshypotheken, ZfIR 2018, 373

\section{Böhringer/Hintzen}

WEG-Novelle 2007, Rpfleger 2007, 353

Bork

Die „kalte Zwangsvollstreckung“ - ein heißes Eisen, ZIP 2013, 2129

\section{Böttcher}

Aktuelle Rechtsprechung zur Zwangsversteigerung im Jahre 2018, ZfIR 2019, 11

\section{Böttcher}

Schuldner-, Bieter-, und Ersteherzahlungen im Versteigerungsverfahren, ZfIR 2007, 597

\section{Böttcher}

Veräußerung von Immobilien während eines Zwangsversteigerungsund Zwangsverwaltungsverfahrens, ZfIR 2010, 521

\section{Brandau/Draga}

Die Entrichtungspflicht der Einkommensteuer für den Zwangsverwalter, ZfIR 2016, 700

\section{Brandau/Strob/Bubrfeind}

Die Mietsicherheit in der Zwangsverwaltung, ZfIR 2015, 646

\section{Brändle}

Energielieferung in der Zwangsverwaltung, ZfIR 2016, 729

\section{Bräuer}

Die zwangsversteigerungsfeste Erbbauzinsreallast, Rpfleger 2004, 401

\section{Burbulla}

Baulasten: Häufige Problemfelder in der Praxis und der Zwangsversteigerung, ZfIR 2018, 717

\section{Clemente}

Die in der Versteigerung bestehen bleibende Grundschuld. Irrtümer und Enttäuschungen von Angestellten und Erstehern, ZfIR 2019, 325

\section{Commans}

Die Zwangsversteigerung von Wohnungseigentum wegen Wohngeldrückständen und die Problematik des Einheitswertbescheids, ZfIR 2009, 489 


\section{Cranshaw}

Effizientes Zwangsverwaltungs- und Zwangsversteigerungsmanagement Perspektiven aus Gläubigersicht, ZfIR 2013, 345 und 393

\section{Cranshaw}

Schnittstellen der Zwangsverwaltung zum Insolvenzverfahren und zur Sicherungsverwaltung, ZfIR 2019, 425

\section{Cranshaw/Gietl}

Titel oder Klausel - Räumung gegen Mit,,besitzer“ nach \93 ZVG, ZfIR 2010, 753

\section{Cranshaw/Welsch}

Kalte bzw. stille Zwangsverwaltung - Vorteile und Zweifelsfragen, DZWIR 2017, 101

\section{Depré}

Die stille Zwangsverwaltung - Zweckmäßigkeit und Grenzen, ZfIR 2017, 1

\section{Depré}

Die Zeitvergütung in der Zwangsverwaltung - Richtlinien für die Praxis, ZfIR 2008, 49

\section{Depré}

Eine Antragsrücknahme im Zwangsversteigerungs- oder

Zwangsverwaltungsverfahren erfordert eine konstitutive Entscheidung des Vollstreckungsgerichts, ZfIR 2008, 841

\section{Derleder}

Die neue Sicherungsgrundschuld, ZIP 2009, 2221

\section{Derleder}

Die Realisierung des Vorrangs des Hausgeldes bei der Zwangsversteigerung und Zwangsverwaltung von Eigentumswohnungen, ZWE 2008, 13

\section{Drasdo}

Der Irrtum des BFH, NZI 2019, 793

\section{Drasdo}

Die Abrechnung des nach $\ 94$ ZVG gerichtlich bestellten Verwalters, NZI 2019, 208

\section{Drasdo}

Die Abrechnungspflicht des Zwangsverwalters gegenüber dem Ersteher, NZI 2014, 97

\section{Drasdo}

Die Auswirkungen der WEG-Reform auf die Stellung des Zwangsverwalters, $\mathrm{ZInsO} 2009,862$

\section{Drasdo}

Die Bestellung des Wohnungseigentumsverwalters im Rahmen der Zwangsverwaltung, NZI 2015, 1 
Drasdo

Die gerichtliche Verwaltung gem. \94 ZVG, NZI 2014, 846

Drasdo

Die Höchstpersönlichkeit der Amtsstellung des Zwangsverwalters, NZI 2015, 393

Drasdo

Die Mitteilungspflichten des Zwangsverwalters, NZI 2013, 614

Drasdo

Die Verwaltung im Rahmen des $\ 25$ ZVG, ZfIR 2015, 423

Drasdo

Die Wohnraumüberlassung an den Zwangsverwaltungsschuldner, ZfIR 2013, 839

Drasdo

Die Zwangsverwaltung von Wohnungseigentum, ZWE 2006, 68

Drasdo

Unterlassene Vermietung durch den Zwangsverwalter, NZI 2016, 937

Drasdo

Unzureichende Masse - Zahlungspflichten des Zwangsverwalters, NZI 2017, 918

Drasdo

Zugriffsmöglichkeiten des Zwangsverwalters bei besonderen mietrechtlichen Situationen, NZI 2016, 246

Engels

Altlasten in der Zwangsversteigerung und Zwangsverwaltung, Rpfleger 2010, 557

Engels

Zwangsverwaltung und Einkommensteuer, Rpfleger 2015, 525

Ertle

Die Sequestration nach $\ 25$ ZVG, ZfIR 2016, 385

Ertle

Die Vertretung „unbekannter Beteiligter“ im Zwangsversteigerungsverfahren, ZfIR 2018, 815

Ertle

Die Vertretung vor dem Vollstreckungsgericht. Die Anmeldung von Mietverträgen, ZfIR 2013, 9

Ertle/Schmidberger

Suizid - Vollstreckung zwischen Wunsch und Wirklichkeit, ZfIR 2016, 609

Fischer

Der Grundschuldgläubiger und seine Pflicht, die Rechtsprechung des BGH ständig zu überwachen, ZInsO 2012, 1493. 


\section{Fischer}

Der Zwangsverwalter und $\ 135$ Abs. 3 InsO - gesellschaftsinterne

Nutzungsverhältnisse, ZfIR 2010, 312

\section{Fischer/Knees}

Zum Umgang des Grundpfandrechtsgläubigers mit $\ 135$ Abs. 3 InsO, ZInsO 2009, 745

\section{Fischer/Lorenz/Biederbeck}

Die Erstellung von Gutachten bei Zwangsversteigerungen, Rpfleger 2002, 337

\section{Fischinger}

Aktuelle Fragen der Zwangshypothek, WM 2009, 637

\section{Goldbach}

Der Verkündungstermin im Zwangsversteigerungsverfahren, ZfIR 2013, 793

\section{Goldbach}

Die öffentliche Hand in Zwangsverwaltung und Zwangsversteigerung, ZfIR 2017, 384

\section{Goldbach}

Erneuerbare Energien im Zwangsversteigerungsverfahren, ZfIR 2014, 37

\section{Goldbach}

Rückgewähransprüche bei der Immobilienverwertung, ZfIR 2019, 45

\section{Groß}

Das Eigengebot des Terminvertreters ist wirksam, Rpfleger 2008, 545

\section{Haarmeyer}

Die neue Vergütung in der Zwangsverwaltung - ein erster Überblick, ZInsO 2004, 18

\section{Hartung}

Grundsteuer und weitere öffentliche Grundstückslasten in der Zwangsverwaltung, Rpfleger 2013, 661

\section{Hartung}

Mietkaution und Zwangsverwaltung, NZI 2014, 739

\section{Hasselblatt}

Scheingebote im Zwangsversteigerungsverfahren oder: Werden

Gläubigervertreter noch ernst genommen?, NJW 2006, 1320

\section{Hawelka}

Die problematische Inbesitznahme bei der Zwangsverwaltung, ZfIR 2005, 14

\section{Hintzen}

Beschlagnahmewirkung nach Antragsrücknahme in der Zwangsverwaltung, Rpfleger 2009, 68 


\section{Hintzen}

Das Hausgeldinkasso - 2. Teil: Die Vollstreckung - Zwangsversteigerung, Zwangsverwaltung, Insolvenz, ZWE 2018, 249

\section{Hintzen}

Die Entwicklung im Zwangsversteigerungs- und Zwangsverwaltungsrecht seit 2001, Rpfleger 2004, 69

\section{Hintzen}

Grundstücksverwertung durch den Treuhänder in der Verbraucherinsolvenz, ZInsO 2004, 713

\section{Hintzen}

Widerspruch gegen den Teilungsplan. Der Teilungsplan als Entscheidung - BGH versus Praxis, Rpfleger 2016, 258

\section{Hintzen/Alff}

Änderungen des ZVG aufgrund des Zweiten JuModG, Rpfleger 2007, 233

\section{Hintzen/Alff}

Die neue Zwangsverwalterverordnung, Rpfleger 2004, 129

\section{Jacoby}

Insolvenz- und Zwangsverwaltung. Gleichklang und Dissonanzen?!, ZfIR 2017, 685

\section{Jähne/Witte}

Pflicht des Sicherungsnehmers einer Grundschuld zur Geltendmachung von Grundschuldzinsen, Rpfleger 2012, 473

\section{Jobannsen}

Der Schutz des Hypothekengläubigers durch die Feuerversicherung im gestörten Versicherungsverhältnis, NVersZ 2000, 410

\section{Jungmann}

Die einstweilige Einstellung der Zwangsverwaltung im Insolvenzeröffnungsverfahren, NZI 1999, 352

\section{Kablert}

Das BMF-Schreiben vom 3.5.2017 betreffend die ertragssteuerrechtlichen Pflichten des Zwangsverwalters. Überblick und Einordnung für die Praxis, ZfIR 2017, 649

\section{Keller}

Aktuelle Rechtsprechung zur Zwangsverwaltung im Jahre 2008, ZfIR 2009, 385

\section{Keller}

Aktuelle Rechtsprechung zur Zwangsverwaltung im Jahre 2015, ZfIR 2016, 253

\section{Keller}

Altlastensanierung durch den Zwangsverwalter bei Insolvenz des

Grundstückseigentümers, Rpfleger 2010, 568 


\section{Keller}

Die Rechtsprechung zu Fragen der Zwangsverwaltung im Jahre 2006, ZfIR 2007, 377, 380

\section{Kesseler}

Verfahrensbedingte Risiken des Finanzierungsgläubigers des Erstehers im Zwangsversteigerungsverfahren, WM 2005, 1299

Kirchbof

Zwei Jahre Insolvenzordnung - ein Rückblick, ZInsO 2001, 1

Klawikowski

Die Auswirkungen der Grundstücksversteigerung auf Miet- und Pachtverhältnisse, Rpfleger 1997, 418

Klawikowski

Die Grundstücksversteigerung bei Vor- und Nacherbschaft, Rpfleger 1998, 100

Klawikowski

Probleme des Erstehers nach der Zwangsversteigerung, Rpfleger 2014, 236

Klawikowski

Rückzahlung von Vorschüssen aus der Zwangsverwaltung, Rpfleger 2013, 483

Klawikowski

Schadensfälle in der Grundstücksversteigerung, Rpfleger 2005, 341

Klawikowski

Vertretung von Beteiligten und Bietern im Zwangsversteigerungsverfahren, Rpfleger 2008, 404

Klein

Einstweilige Einstellung der gerichtlichen Zwangsverwaltung in Massegrundstücke auch auf Antrag des vorläufigen Insolvenzverwalters?, $\mathrm{ZInsO} 2002,1065$

Klühs

Die Pflicht des Zwangsverwalters zur Abrechnung von Betriebskosten und Auszahlung entsprechender Mieterguthaben, Rpfleger 2006, 640

Knees

Die Bank als Gläubiger/Schuldner des Grundschuldrückgewähranspruchs, ZIP 2018, 1055

Knees

Die Bank als Grundpfandrechtsgläubiger in der Unternehmensinsolvenz, ZIP 2001, 1568

\section{Kummer}

Systematische und Interessen abwägende Aufhebung der Zwangsverwaltung nach Zuschlag, ZfIR 2014, 767 
Lwowski/Tetzlaff

Altlasten in der Insolvenz - Freigabe, Insolvenzplan und parallele Zwangsverwaltungsverfahren, NZI 2004, 225

Mayer

Der Zwangsverwalter, die Mietkaution und der BGH, Rpfleger 2006, 175 Mayer

Die Beschlagnahme in der Zwangsverwaltung, Rpfleger 2009, 287

Mayer

Zwangsverwaltung - Zuschlag und dennoch kein Ende!, ZfIR 2013, 51

Meerhoff

Der Zuschlagsbeschluss als Vollstreckungstitel, ZfIR 2018, 93

Meerboff

Die GbR als Bieterin im Zwangsversteigerungstermin, ZfIR 2015, 518

Meerboff

Festsetzung eines Zuzahlungsbetrags gem. \51 ZVG, ZfIR 2013, 494

Meerboff

Gebäudeversicherung in der Immobiliarzwangsvollstreckung, ZfIR 2017, 180

Meerhoff

Probleme bei der Mitversteigerung von Grundstückszubehör,

ZfIR 2016, 556

Meerboff

Rangverlust und späte Anmeldung eines Wertersatzes, ZfIR 2016, 263

Meerboff

Verfahrenseinstellung gem. \75 ZVG, ZfIR 2013, 796

Meerboff

Zur Immobiliarzwangsvollstreckung bei Vor- und Nacherbschaft,

ZfIR 2017, 308

Meerhoff

Zwangsversteigerungsverfahren ohne Schuldner, ZfIR 2015, 704

Mengwasser

Die Nebenkostenabgrenzung zwischen Zwangsverwalter und Ersteher, ZfIR 2012, 495

Mette

Interessenkollision eines (Instituts-)Zwangsverwalters, Rpfleger 2003, 170 Metz

Zur Anfechtbarkeit der Verkehrswertfestsetzung im Zwangsversteigerungsverfahren von Privathotels aus verfassungsrechtlicher Sicht, Rpfleger 2010, 13

Milger

Miete und Kaution in der Zwangsverwaltung, NJW 2011, 1249 


\section{Mönning/Zimmermann}

Die Einstellungsanträge des Insolvenzverwalters gem.

\S 30 d I, 153 b I ZVG im eröffneten Insolvenzverfahren, NZI 2008, 134

\section{Nedden-Boeger}

Fortdauer der Zwangsverwaltung nach Aufhebung wegen Zuschlags, ZfIR 2014, 577

\section{Nettersheim}

Anforderungen der Rechtsprechung des Bundesverfassungsgerichts an die Auslegung und Anwendung von $\$$ 765a ZPO, ZfIR 2017, 174

Nöll

Insolvenzverwalter vs. Zwangsverwalter: Grenzen haftungsrechtlicher Zuweisung von Zwangsverwaltungserlös an Grundpfandgläubiger, $\mathrm{ZInsO} 2007,1125$

\section{Onusseit}

Zwangsverwaltung und Umsatzsteuer, ZfIR 2005, 265

\section{Onusseit}

Zwangsverwaltung und Umsatzsteuer, ZfIR 2007, 121

Pape

Die Vergütung nach der neuen Zwangsverwalterverordnung, NZI 2004, 187

Piekenbrock

Die Bietsicherheit von Banken: ein Lehrstück missglückter Gesetzgebung, WM 2009, 969.

\section{Rellermeyer}

Der Scheckausstellungszeitraum des $\ 69$ ZVG, Rpfleger 2012, 181

\section{Schlamann}

Das lebenslange Wohnungsrecht im Versteigerungsverfahren, ZInsO 2020, 69

\section{Schmid}

Zwangsversteigerung, Zwangsräumung und Selbstmorddrohung, ZfIR 2014, 838

\section{Schmidberger}

\65 ZVG - eine Vorschrift ohne Bedeutung?, ZfIR 2018, 724

\section{Schmidberger}

Der Besitz und die Immobiliarvollstreckung, Rpfleger 2008, 105

\section{Schmidberger}

Die Einstellung der Zwangsverwaltung, ZfIR 2009, 276

\section{Schmidberger}

Ersteheransprüche aus Nebenkostenabrechnung, ZInsO 2008, 83

\section{Schmidberger}

Mieteinzug in der Zwangsverwaltung, ZfIR 2019, 613 


\section{Schmidberger}

Renaissance der Institutsverwaltung?, ZInsO 2007, 1137

\section{Schmidberger}

Zwang gegen den Verwalter, ZfIR 2014, 6

\section{Schmidberger}

Zwangsverwaltung und Vorschüsse für Hausgeld, ZfIR 2010, 1

Schmidberger/Traub

Das Ende der Zwangsverwaltung, ZfIR 2012, 805

Schmidberger/Traub

Der Widerspruch in der Zwangsverwaltung, Rpfleger 2010, 117

Schmidberger/Traub

Hat $\ 6$ ZVG eine Zukunft?, ZfIR 2016, 339

\section{Schmidt-Räntsch}

Betriebsfortführung in der Zwangsverwaltung, ZInsO 2006, 303

\section{Schmidt-Wudy}

Aktuelle Fragestellungen zu $\int 42$ ZVG unter besonderer Berücksichtigung der Sicht von Bietinteressenten, Rpfleger 2014, 293

\section{Schneider}

Ausgewählte Fragestellungen zur Immobiliarvollstreckung nach der WEG-Novelle 2007, ZfIR 2008, 161

\section{Schneider}

Die verspätete oder unterbliebene Anmeldung von Hausgeldansprüchen in der Zwangsversteigerung eines Wohnungseigentums, ZMR 2018, 119

\section{Schneider}

Einmalige öffentliche Lasten in der Zwangsversteigerung eines Grundstücks, ZfIR 2019, 666

\section{Schneider}

Gewöhnliche Miteigentümer und Wohnungseigentümer als Beteiligte gem. \9 ZVG?, ZfIR 2012, 613

\section{Steffen}

Die Behandlung der „neuen“ Hinterlegungszinsen im Teilungsplan der Zwangsversteigerung, Rpfleger 2011, 360

Steffen

Die Ersteherverwaltung nach $\int 94$ ZVG, ZfIR 2016, 92

\section{Stöber}

Insolvenzverfahren und Vollstreckungs-Zwangsversteigerung, NZI 1998, 105

\section{Stöber}

Verjährte, rückständige und laufende Grundschuldzinsen in der Zwangsversteigerung, MittBayNot 1999, 441 


\section{Storz/Kiderlen}

Der Gesetzgeber, der BGH und die Zwangsversteigerung, NJW 2007, 1846

\section{Strener}

Verzinsung der gemäß $\ 118$ Abs. 1 ZVG übertragenen Forderung und

Neuregelung des Schuldnerverzugs im Fall einer Geldforderung,

Rpfleger 2001, 401

Tetzlaff

Probleme bei der Verwertung von Grundpfandrechten und Grundstücken im Insolvenzverfahren, ZInsO 2004, 521

Tetzlaff

Rechtsprobleme der kalten Zwangsverwaltung, ZfIR 2005, 179

Traub

Die Vertreterbestellung für unbekannte Berechtigte, $\ 135$ ZVG, ZfIR 2015, 823

Traub

Grundstücksrechte im Grundbuch und in der Zwangsversteigerung, ZfIR 2019, 483

\section{Ulrich}

Suizid als sittenwidrige Härte gem. \765a ZPO, Rpfleger 2012, 477

\section{Vonnemann}

Die Abwicklung des Zwangsverwaltungsverfahrens durch den Zwangsverwalter nach Aufhebung der Zwangsverwaltung, Rpfleger 2002, 415

\section{Wedekind}

Zur Schlechterstellung der Wohnungseigentümergemeinschaften im Zwangsverwaltungsverfahren durch die WEG-Reform, ZfIR 2007, 704

Weis

Die Zukunft der Institutsverwaltung nach In-Kraft-Treten der neuen

Zwangsverwalterverordnung, ZInsO 2004, 233

\section{Weis/Ristelhuber}

Die Verwertung von Grundbesitz im Insolvenzverfahren und die Kostenpauschalen für die Insolvenzmasse, ZInsO 2002, 859

\section{Wendlinger}

Der Pflichtenkreis des Zwangsverwalters nach Aufhebung der Zwangsverwaltung, Rpfleger 2009, 544

\section{Wenzel}

Die Rechtsstellung des Grundpfandrechtsgläubigers im Insolvenzverfahren, NZI 1999, 101

\section{Wenzel}

Die Zahlungspflichten des Zwangsverwalters gegenüber der Wohnungseigentümergemeinschaft, ZInsO 2005, 113 


\section{Wipperfürth}

Das Erbbaurecht in der Zwangsversteigerung und im Insolvenzverfahren - ein amorphes Fabelwesen oder kristalline Rechtspraxis?, ZfIR 2019, 784

\section{Witte/Jähne}

Terminswahrnehmung in der Zwangsversteigerung durch Mitarbeiter eines Rechtsanwalts, Rpfleger 2010, 65

\section{Zeiser}

Zwangssicherungshypothek wegen Wohngeldansprüchen nach der WEG-Reform, Rpfleger 2008, 58

\section{Zipperer}

Der Anspruch des Mieters auf Rückerstattung der Kaution - eine Risikobetrachtung für Erwerber und Gläubiger des Vermieters, ZfIR 2007, 388

\section{Zipperer}

Probleme beim Zusammentreffen von Zwangsverwaltung und Insolvenzverwaltung, ZfIR 2011, 385

\section{Zschieschack}

Der Suizid-Einwand im Zwangsverwaltungs- und Zwangsversteigerungsverfahren aus juristischer Sicht, ZfIR 2019, 549 